\title{
Three-Dimensional Echocardiographic Assessment of the Aortic Valve and the Aorta
}

\author{
Aimen Smer ${ }^{1}$, Prakrity Urja ${ }^{2}$, Dixitha Anugula ${ }^{2}$, Subash Dulal $^{3}$, Zeyad Elmarzouky ${ }^{3}$, \\ Edward Gill ${ }^{4}$, and Navin Nanda ${ }^{3}$ \\ ${ }^{1}$ Affiliation not available \\ ${ }^{2}$ Creighton University School of Medicine \\ ${ }^{3}$ University of Alabama at Birmingham \\ ${ }^{4}$ University of Colorado Denver - Anschutz Medical Campus
}

March 3, 2021

\begin{abstract}
We describe the role of three-dimensional echocardiography in the assessment of the aortic valve and the aorta. The manuscript is heavily illustrated with figures and movie clips.
\end{abstract}

\section{Introduction}

Echocardiography is the primary diagnostic imaging modality for the diagnosis and management of patients with aortic valve disease. In most cases, two-dimensional (2D) transthoracic echocardiogram can provide accurate information on the morphology and function of the aortic valve. However, given the complexity of the aortic valve and aortic root anatomy, other advanced imaging modalities such as multislice computer tomography (MSCT), magnetic resonance imaging (MRI) and three-dimensional (3D) echocardiography have been introduced to improve the diagnostic accuracy and guide interventional procedures. Overall, 3D echocardiography has several advantages over two-dimensional (2D) echocardiography, MSCT and MRI and provides accurate assessment of aortic valve and aorta. In this article we discuss the clinical applications of $3 \mathrm{D}$ echocardiography in the evaluation of the aortic valve and the thoracic aorta.

\section{Aortic Valve Anatomy}

The recent revolution in the field of interventional cardiology, particularly percutaneous transcatheter aortic valve interventions (TAVR) has led to better understanding of the complex anatomy of aortic valve apparatus. The aortic valve is composed of three semilunar cusps attached to the aortic root. It is important to understand that the function of the aortic valve depends on the integrity of the aortic root, which refers to the region between the aortic valve junction at the left ventricular outflow tract (LVOT), known as the ventriculo-arterial junction, to the sinotubular junction at the origin of the proximal ascending thoracic aorta. Any anatomical changes of the aortic root can adversely affect the function of the aortic valve. For instance, dilatation of the aortic root and ascending aorta can lead to aortic regurgitation despite intact aortic valve leaflets.

The main advantage of 3D echocardiography over conventional 2D modality, its ability to acquire the entire aortic valve and surrounding structures in 3D dataset, which allows visualization of the aortic valve in en face view from different perspectives and facilitates cropping in multiplanar reconstruction mode. The use of 3D echocardiography can provide accurate assessment of the aortic valve morphology and eliminates any geometric assumptions of the LVOT, aortic annulus and root measurements. In general, transthoracic 3D 
imaging of the aortic valve remains challenging in many cases, yet is feasible and the image quality has improved dramatically with newer 3D echocardiography machines. Using transthoracic 3D matrix-array transducer, the aortic valve can be imaged from both parasternal and apical views. For anatomic assessment of the aortic valve, live 3D or 3D zoom acquisition modes are the preferred imaging modes. Adding 3D color Doppler is used to assess for aortic regurgitation and cusp integrity. Suboptimal 3D transthoracic echocardiography (TTE) images usually occur in patients with poor acoustic windows or heavily calcified aortic valve. In these cases, the use of 3D transesophageal echocardiography (TEE) can provide better image quality with superior spatial resolution and the first report describing its utility in planimetry of the aortic valve orifice area in a patient with aortic stenosis was published as early as 1994 (Figure 1). ${ }^{6}$ Clinical live/real time 3DTEE was first introduced several years later in $2007 .{ }^{7}$ Transthoracic and transesophageal 3D dataset of the aortic valve can be processed and displayed from both aortic and ventricular perspectives, which improves the diagnostic accuracy and helps guide interventional procedures. The use of multiplanar reconstruction mode is very useful in obtaining accurate measurements of the aortic valve area, aortic annulus dimensions, and aortic root size when planning for TAVI or other interventional procedures.

\section{Congenital Aortic Valve Abnormalities}

There are several congenital malformations of the aortic valve which include unicuspid, bicuspid and quadricuspid valves. Bicuspid aortic valve is the most common congenital abnormality of the aortic valve affecting $2 \%$ of the general population. Although standard $2 \mathrm{D}$ echocardiography can accurately delineate the aortic valve morphology in most cases as tricuspid versus bicuspid, the diagnosis can be difficult in calcified aortic valves and in cases of bicuspid aortic valve when a raphe that mimics a commissure. The abi ${ }^{1}$ ity of $3 \mathrm{D}$ echocardiography to visualize the aortic valve in en face view from the aorta provides direct visualization of the aortic valve cusp margins and commissures (Figure 2, Movie 1). The diagnostic accuracy of 3D TEE for bicuspid aortic valve has been validated against anatomic examination of autopsy specimens.

\section{Aortic Stenosis}

Standard 2D and Doppler transthoracic echocardiography remains the primary diagnostic tool for the diagnosis of aortic stenosis using pressure gradients and the continuity equation method. It is important to understand that both methods are flow dependent and indirect estimate of the aortic valve area. There are several limitations to the use of continuity equation to calculate the aortic valve area because it assumes that the LVOT is circular in geometry, which is not true in many cases. In fact, the coronal measurement of the LVOT is the smaller of the LVOT diameters compared to the sagittal. A narrow LVOT also overestimates the severity of aortic stenosis when using the continuity equation. In addition, obtaining the peak Doppler velocity across the aortic valve can be challenging, which may lead to underestimation of the aortic stenosis, whereas overestimation can occur in cases of significant pressure recovery phenomenon. The use of 3D echocardiography either from a transthoracic or transesophageal approach can overcome these limitations and has several advantages over 2D imaging. For instance, the ability of 3D echocardiography to visualize the aortic valve and annulus from different angles allows for accurate planimetry of both the aortic valve and LVOT. Using multiplanar reconstruction mode, 3D datasets of the aortic valve allows cropping to the tips of the aortic valve leaflets for precise planimetry and calculate the aortic valve area (Movie 2). ${ }^{11}$ It also can assess individualized leaflets motion and localize the site of obstruction in patients with subvalvular or supravalvular obstruction.

In addition, 3D echocardiography can be very valuable in the assessment of aortic valve stenosis in certain clinical scenarios when Doppler measurements are inaccurate due to overestimated or underestimated Doppler velocities across the aortic valve. For example, in patients with hypertrophic obstructive cardiomyopathy and high LVOT gradient and in patients with suspected severe low-flow low-gradient aortic stenosis (stage D disease) undergoing low dose Dobutamine challenge, 3D TEE can supplement 2D echocardiography by accurate planimetry of the aortic valve area and precise sizing of the LVOT regardless of the Doppler velocities. Accurate sizing of the LVOT is not only important for quantifying the severity of aortic stenosis, but also for proper annulus sizing for patients undergoing TAVI. However, it is important to understand that $3 \mathrm{D}$ aortic valve planimetry can be limited by three main factors, very low stroke volume which may fail to open 
the aortic valve fully leading to overestimation of the degree of stenosis, lower temporal resolution that may miss the maximal systolic opening of the aortic leaflets, and reverberations artifact of heavily calcified aortic valve leaflets making accurate planimetry of the orifice area difficult to trace. 3DTTE is also useful to assess patients with aortic stenosis who also have another lesion in tandem such as subvalvularor supravalvular stenosis by separate planimetry of the two lesions and determining stenosis at each level. In fact, subvalvular membranes are particularly well imaged by 3D echocardiography. Standard continuous wave Doppler using $2 \mathrm{D}$ echocardiography will give the maximum velocity across both lesions with no differentiation of severity at individual levels (Figure 3A-C). ${ }^{13}$

\section{Aortic Regurgitation}

Aortic regurgitation can arise from either pathological abnormality of the aortic valve leaflets (congenital, degenerative, or infectious) or primary dilatation of the aortic root and ascending thoracic aorta. Identifying the etiology and severity of aortic regurgitation is crucial for decision making regarding the choice and the timing of aortic valve intervention. Therefore, it is important to assess the aortic valve apparatus, ascending aorta, and left ventricle to decide on the type of intervention needed. Similar to aortic stenosis, transthoracic echocardiography is usually sufficient to diagnosis and assess the severity of aortic regurgitation. Once a significant aortic regurgitation is identified by Doppler echocardiography assessing the aortic regurgitation severity, etiology, aortic root dimensions, and left ventricular size and function is essential for comprehensive evaluation. However, both qualitative and quantitative Doppler assessment of aortic regurgitation including pressure half time, vena contracta width, proximal isovelocity surface area, regurgitant volume and fraction suffer several limitations due hemodynamic changes, eccentric jets and inherited geometric assumption that the regurgitant orifice is circular, which is not the case in most cases. Adding TEE and 3D echocardiography can provide more detailed assessment of the aortic regurgitation and aortic valve morphology and function. $3 \mathrm{D}$ echocardiography can reliably identify the mechanism of aortic regurgitation and with the complementary use of 3D color mode provides important diagnostic information about the regurgitant jets size, location, and severity. In addition, full-volume 3D mode can be very useful for accurate quantification of the left ventricular size and function, an important echocardiographic criterion for surgical referral in patients with severe chronic aortic regurgitation.

The recent advances in 3D technology allows for volumetric assessment of the regurgitant volume by measuring the left and right ventricular stroke volumes with strong correlation with other cardiac imaging modalities.In the setting of good 2D image quality, the use of 3D stroke volume measurements eliminate the interobserver variation, geometric assumption of the LVOT size and ventricular foreshortening.

Another unique advantage of 3D over standard 2D echocardiography is the ability to measure the vena contracta area (VCA). Adding 3D color Doppler to live 3D of the aortic valve allows visualization of the regurgitant jet. In a multiplanar reconstruction model, 3D color dataset can be cropped from either the aortic or left ventricular perspective to the level of the narrowest regurgitant jet (vena contracta) in a perpendicular plane to measure the VCA in early diastole (Figure 4, Movie 3). ${ }^{24}$ The VCA can be multiplied by the velocity time integral of the aortic regurgitant jet to calculate the regurgitant volume. 3D measurement of VCA is simple and correlates very well with MRI method. ${ }^{25}$ Noteworthy, the incremental value of 3D echocardiography is invaluable in the evaluation of patients with prosthetic aortic valve regurgitation. The use of color 3D TEE allows for accurate assessment of paravalvular regurgitation site, size, and severity. In addition, live 3D TEE is used to guide percutaneous paravalvular leak closure (Figure 5, Movie 4).

\section{Aortic Masses}

Compared to 2D echocardiography, 3D imaging can provide more accurate diagnostic information regarding the site, size, shape, and number of cardiac masses.

As explained in Figure 6, 2D echo may underestimate the size of a mass lesion unlike 3D echo which will capture the full extent of the mass enabling more accurate measurements. Also, 3D echo permits assessment of volume of any mass involving the aortic valve or aorta which provides superior characterization as compared to mere dimension measurements. 3D TEE also allows en face view of the aortic valve with superb image 
quality and high spatial resolution, which helps identify the presence of any abnormal masses or tumors on the aortic valve leaflets such as thrombus, vegetation, Lambl's excrescences, thickened nodules of Arantius, and papillary fibroelastoma (Figure $7^{29}$, Movies $5,6 \mathrm{~A}$ and $\mathrm{B}^{29}$ and $7^{31}$ ). 3D TEE has also shown incremental value over 2D TEE in the assessment of aortic atherosclerotic ulcers and thrombi (Movies 8 A-D) ${ }^{32}$

\section{Thoracic Aorta}

The aorta is a complex tubular structure divided into five segments: aortic root, tubular portion of the ascending aorta, aortic arch, descending thoracic aorta and abdominal aorta. Various imaging modalities such as echocardiography, MSCT and MRI have been used to evaluate the aorta. Each has it is own advantages and disadvantages. Overall, conventional 2D echocardiography is used to evaluate the aortic root, proximal ascending aorta, and aortic arch dimensions. 3D echocardiography can improve these measurements by identifying real maximum diameters of each part. The best possible 3D images of the ascending aorta can be obtained from the parasternal long-axis view. A recent 3DTTE study has shown visualization of longer segments of the ascending aorta utilizing the right parasternal approach compared to 2DTTE. In addition, right parasternal examination views the right pulmonary artery behind the ascending aorta unlike the left parasternal approach which most often images the superior vena cava rather than the pulmonary artery posterior to the aorta (Figure $8 \mathrm{~A}-\mathrm{C}$ ). ${ }^{34}$ The trunk/right pulmonary artery often serves not only as a landmark to divide the ascending aorta into proximal and distal segments and but also to measure the mid ascending aorta diameter by MSCT. Suprasternal view may identify aortic arch. The use of 3D echocardiography has become more relevant after the introduction of TAVI. Planning for TAVI requires precise aortic annulus measurements for appropriate valve sizing. 3D measurements of the aortic valve area, annulus, root dimensions and annulus distance to the coronary ostia have been validated against MSCT and MRI. Another major advantage of 3D TEE compared to 2D imaging is guidance during TAVI procedures to assist with appropriate device positioning and assessment of procedural success and identifying any potential complications such as paravalvular leak.

Among patients with thoracic aneurysm and dissection, 3D TEE has greater accuracy than 2D TEE in identifying the type and location of aneurysm as well as the dissection. ${ }^{38}$ Our lab showed that in a study of 67 patients with aortic disease, 20 with aortic aneurysm without dissection, 21 with aortic aneurysm and dissection, and 26 with aortic dissection without aneurysm, the use of live 3D TEE increased the level of confidence in the diagnosis and allows the localization of the rupture site (Figures 9, 10 A-D, Movies 9 A-C). ${ }^{39}$ The use of 3D TEE can easily distinguish linear artifact occasional seen within the aorta from a true dissection flap, which will appear like a curvilinear sheet rather than a thin linear echo density (Figure $11 \mathrm{~A}$ and $\mathrm{B}$, Movies $10 \mathrm{~A}$ and $\mathrm{B}) .{ }^{40}$

\section{Conclusion}

Over the last decade, the role of 3D echocardiography in the evaluation of aortic valve disease and aorta has been growing dramatically due to the many advantages of 3D technology in both the diagnostic and therapeutic aspects. However, 3D imaging remains complementary to 2D echocardiography and it relies on good quality 2D images. The lower temporal resolution, reverberations artifact of a heavily calcified valve, and dropout artifact due to thick leaflets are the main challenges to current 3D technology.

\section{Acknowledgments}

None.

\section{Financial support and sponsorship}

None.

\section{Conflicts of interest}

All authors declared that there are no conflicts of interest.

\section{References}


1. Zoghbi WA, Adams D, Bonow RO, et al. Recommendations for noninvasive evaluation of native valvular regurgitation: A report from the american society of echocardiography developed in collaboration with the society for cardiovascular magnetic resonance. J Am Soc Echocardiogr. 2017;30(4):303-371.

2. Tuzcu EM, Kapadia SR, Schoenhagen P. Multimodality quantitative imaging of aortic root for transcatheter aortic valve implantation: More complex than it appears. J Am Coll Cardiol. 2010;55(3):195-197.

3. Ho SY. Structure and anatomy of the aortic root. Eur J Echocardiogr. 2009;10(1):i3-10.

4. Otani K, Takeuchi M, Kaku K, et al. Assessment of the aortic root using real-time 3D transesophageal echocardiography. Circ J. 2010;74(12):2649-2657.

5. Muraru D, Badano LP, Vannan M, et al. Assessment of aortic valve complex by three-dimensional echocardiography: A framework for its effective application in clinical practice. Eur Heart J Cardiovasc Imaging. 2012;13(7):541-555.

6. Nanda NC, Roychoudhury D, Chung SM, et al. Quantitative assessment of normal and stenotic aortic valve using transoesophageal three dimensional echocardiography. Echocardiography. 1994;11:617-625.

7. Pothineni KR, Inamdar V, Miller AP, Nanda NC, et al.Initial experience with live/real time threedimensional transesophageal echocardiography. Echocardiography 2007;24:1099-1104

8. Rong LQ, Hameed I, Salemi A, et al. Three-dimensional echocardiography for transcatheter aortic valve replacement sizing: A systematic review and meta-analysis. J Am Heart Assoc. 2019;8(19):e013463.

9. Siu SC, Silversides CK. Bicuspid aortic valve disease. J Am Coll Cardiol. 2010;55(25):2789-2800.

10. Espinola-Zavaleta N, Munoz-Castellanos L, Attie F, et al. Anatomic three-dimensional echocardiographic correlation of bicuspid aortic valve. J Am Soc Echocardiogr. 2003;16(1):46-53.

11. Nanda NC, Hsiung MC, Miller AP, et al. Live/Real Time 3D Echocardiography. Oxford, UK: WileyBlackwell; 2010.

12. Agrawal GG, Nanda NC, Htay T, et al. Live three-dimensional transthoracic echocardiographic identification of discrete subaortic membranous stenosis. Echocardiography. 2003;20(7):617-619.

13. Rajdev S, Nanda NC, Patel AU, et al. Live/real-time three-dimensional transthoracic echocardiographic assessment of combined valvar and supravalvar aortic stenosis. Am J Geriatr Cardiol. 2006;15(3):188-190.

14. Zog WA, Adams D, Bonow RO, et al. Recommendations for noninvasive evaluation of native valvular regurgitation: A report from the american society of echocardiography developed in collaboration with the society for cardiovascular magnetic resonance. J Am Soc Echocardiogr. 2017;30(4):303-371.

15. Cai Q, Ahmad M. Three-dimensional echocardiography in valvular heart disease. Echocardiography. 2012;29(1):88-97.

16. Roldan CA, Qualls CR, Sopko KS, et al. Transthoracic versus transesophageal echocardiography for detection of libman-sacks endocarditis: A randomized controlled study. J Rheumatol. 2008;35(2):224-229.

17. Reynolds HR, Jagen MA, Tunick PA, et al. Sensitivity of transthoracic versus transesophageal echocardiography for the detection of native valve vegetations in the modern era. J Am Soc Echocardiogr. 2003;16(1):67-70.

18. Steeds RP, Myerson SG. Imaging assessment of mitral and aortic regurgitation: Current state of the art. Heart. 2020;106(22):1769-1776.

19. Nishimura RA, Otto CM, Bonow RO, et al. 2014 AHA/ACC guideline for the management of patients with valvular heart disease: A report of the american college of cardiology/american heart association task force on practice guidelines. J Am Coll Cardiol. 2014;63(22):e57-185. 
20. Namisaki H, Nabeshima Y, Kitano T, et al. Prognostic value of the right ventricular ejection fraction, assessed by fully automated three-dimensional echocardiography: A direct comparison of analyses using right ventricular-focused views versus apical four-chamber views. J Am Soc Echocardiogr. 2021;34(2):117-126.

21. Muraru D, Spadotto V, Cecchetto A, et al. New speckle-tracking algorithm for right ventricular volume analysis from three-dimensional echocardiographic data sets: Validation with cardiac magnetic resonance and comparison with the previous analysis tool. Eur Heart J Cardiovasc Imaging. 2016;17(11):1279-1289.

22. Stoebe S, Metze M, Jurisch D, et al. Analysis of chronic aortic regurgitation by 2D and 3D echocardiography and cardiac MRI. Echo Res Pract. 2018;5(2):51-62.

23. Dorosz JL, Lezotte DC, Weitzenkamp DA, et al. Performance of 3-dimensional echocardiography in measuring left ventricular volumes and ejection fraction: A systematic review and meta-analysis. J Am Coll Cardiol. 2012;59(20):1799-1808.

24. Fang L, Hsiung MC, Miller AP, et al. Assessment of aortic regurgitation by live three-dimensional transthoracic echocardiographic measurements of vena contracta area: Usefulness and validation. Echocardiography. 2005;22(9):775-781.

25. Perez de Isla L, Zamorano J, Fernandez-Golfin C, et al. 3D color-doppler echocardiography and chronic aortic regurgitation: A novel approach for severity assessment. Int J Cardiol. 2013;166(3):640-645.

26. Kinno M, Raissi SR, Olson KA, et al. Three-dimensional echocardiography in the evaluation and management of paravalvular regurgitation. Echocardiography. 2018;35(12):2056-2070.

27. Zamorano JL, Badano LP, Bruce C, et al. EAE/ASE recommendations for the use of echocardiography in new transcatheter interventions for valvular heart disease. J Am Soc Echocardiogr. 2011;24(9):937-965.

28. Alli OO, Hsiung MC, Guvenc T, et al. Incremental Value of Three-Dimensional Transesophageal Echocardiography over the Two-Dimensional Technique in Percutaneous Closure of Aortic Paraprosthetic Regurgitation. Echocardiography. 2014:31:1154-1158

29. Dumaswala B, Dumaswala K, Hsiung MC, et al. Incremental value of three-dimensional transesophageal echocardiography over two-dimensional transesophageal echocardiography in the assessment of Lambl's excrescences and nodules of Arantius on the aortic valve. Echocardiography. 2013;30(8):967-975.

30. Nanda NC. Incremental value of three-dimensional echocardiography over transesophageal multiplane two-dimensional echocardiography in qualitative and quantitative assessment of cardiac masses and defects. Echocardiography. 1995;12:619-28.

31. Hansalia S, Biswas M, Dutta R,et al. The value of live/real time three-dimensional transesophageal echocardiography in the assessment of valvular vegetations. Echocardiography.2009;26:1264-1273

32. Elsayed M, Bulur S, Kalla A, et al. Incremental value of live/real time three-dimensional transesophageal echocardiography over the two-dimensional technique in the assessment of aortic atherosclerotic thrombi and ulcers. Echocardiography. 2016;33(8):1234-1238.

33. Goldstein SA, Evangelista A, Abbara S, et al. Multimodality imaging of diseases of the thoracic aorta in adults: From the American Society of Echocardiography and the European Association of Cardiovascular Imaging: Endorsed by the Society of Cardiovascular Computed Tomography and Society for Cardiovascular Magnetic Resonance. J Am Soc Echocardiogr. 2015;28(2):119-182.

34. Akdogan RE, Srialluri S, Abdelsalam MGA, et al. Incremental value of right parasternal transthoracic echocardiographic examination over the left parasternal approach in assessing ascending aorta size: Additional benefit of three-dimensional echocardiography. Echocardiography. 2019;36:1306-1314.

35. Altiok E, Koos R, Schroder J, et al. Comparison of two-dimensional and three-dimensional imaging techniques for measurement of aortic annulus diameters before transcatheter aortic valve implantation. Heart. 2011;97(19):1578-1584. 
36. Paelinck BP, Van Herck PL, Rodrigus I, et al. Comparison of magnetic resonance imaging of aortic valve stenosis and aortic root to multimodality imaging for selection of transcatheter aortic valve implantation candidates. Am J Cardiol. 2011;108(1):92-98.

37. Joshi D, Bicer EI, Donmez C, et al. Incremental value of live/real time three-dimensional transesophageal echocardiography over the two-dimensional technique in the assessment of aortic aneurysm and dissection. Echocardiography. 2012;29(5):620-630.

38. Sasaki S, Watanabe H, Shibayama K, et al. Three-dimensional transesophageal echocardiographic evaluation of coronary involvement in patients with acute type A aortic dissection. J Am Soc Echocardiogr. 2013;26(8):837-845.

39. Htay T, Nanda NC, Agrawal G, et al. Live three-dimensional transthoracic echocardiographic assessment of aortic dissection. Echocardiography. 2003;20(6):573-577.

stylefix

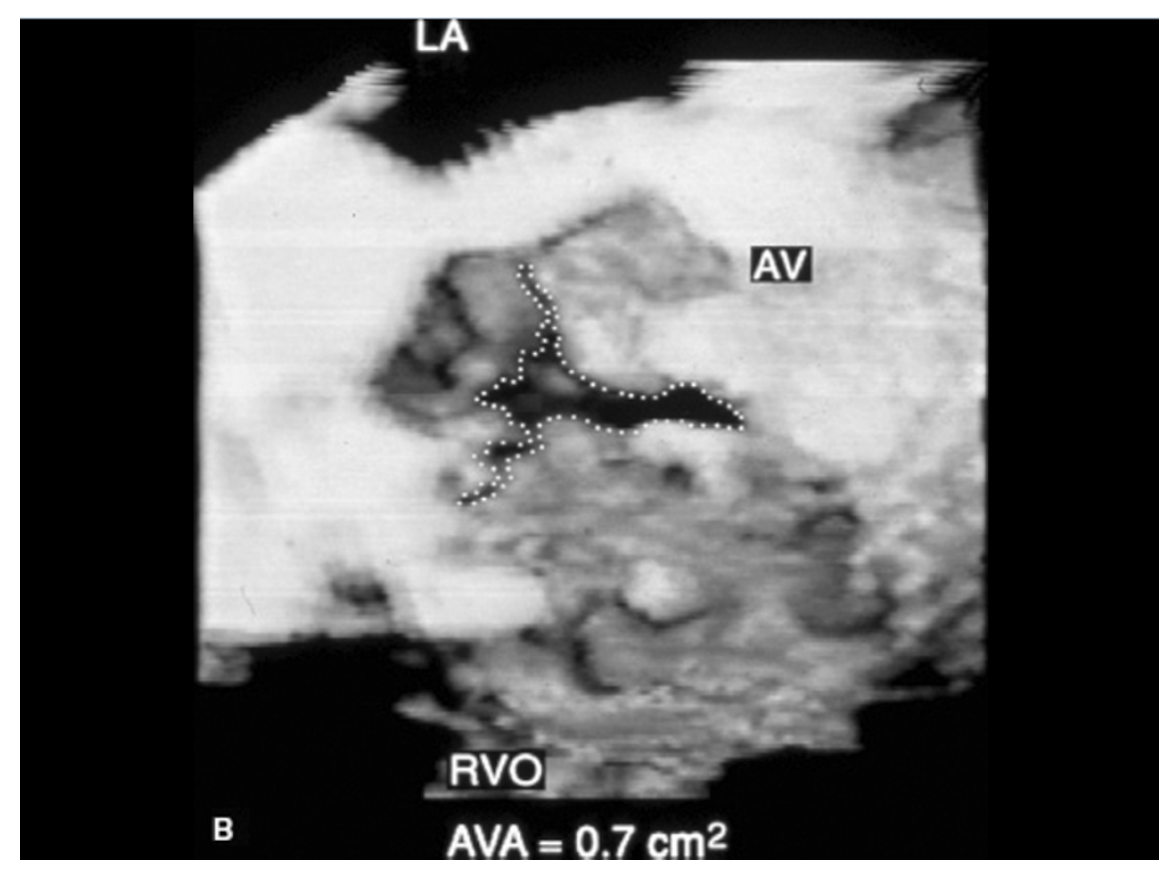



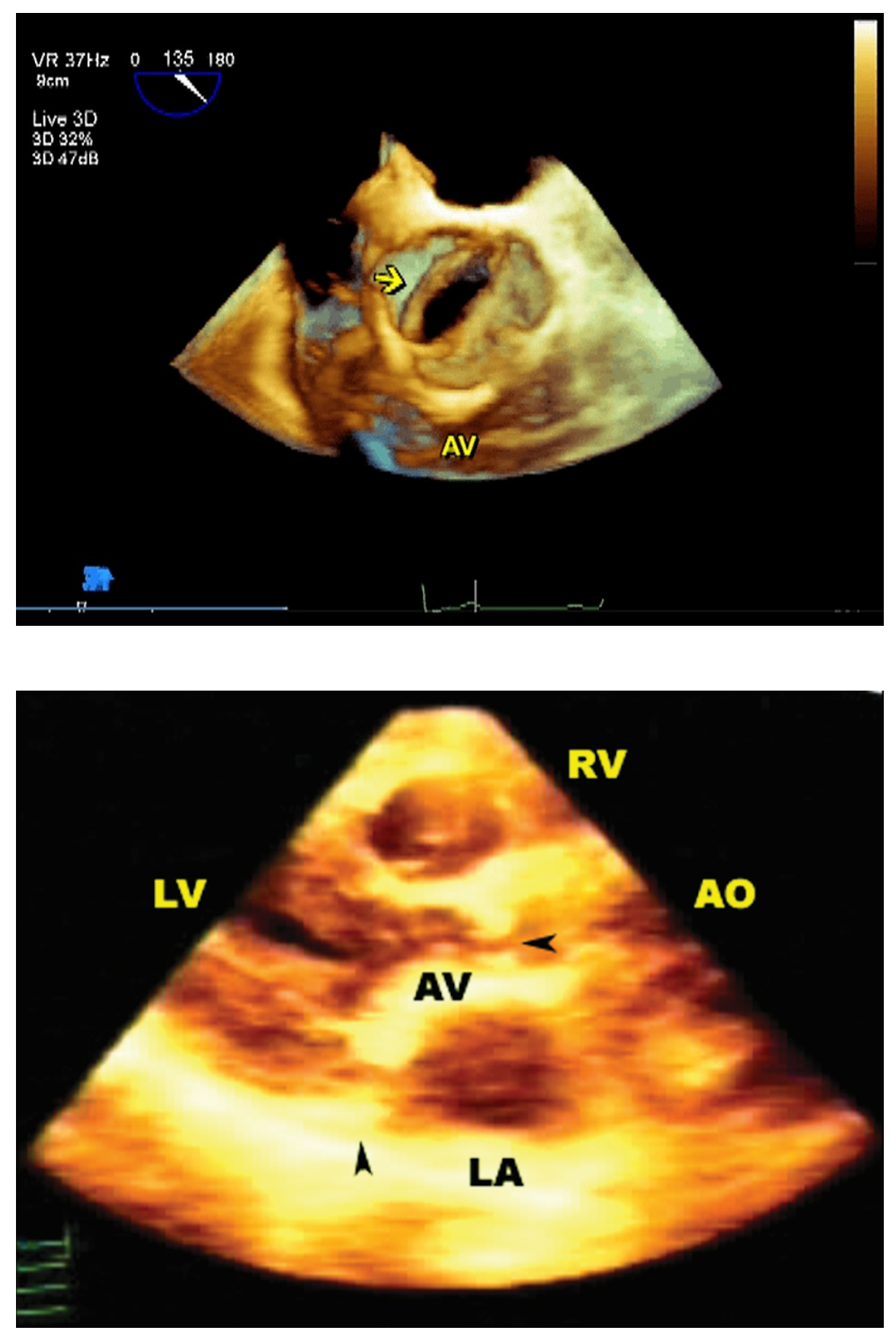

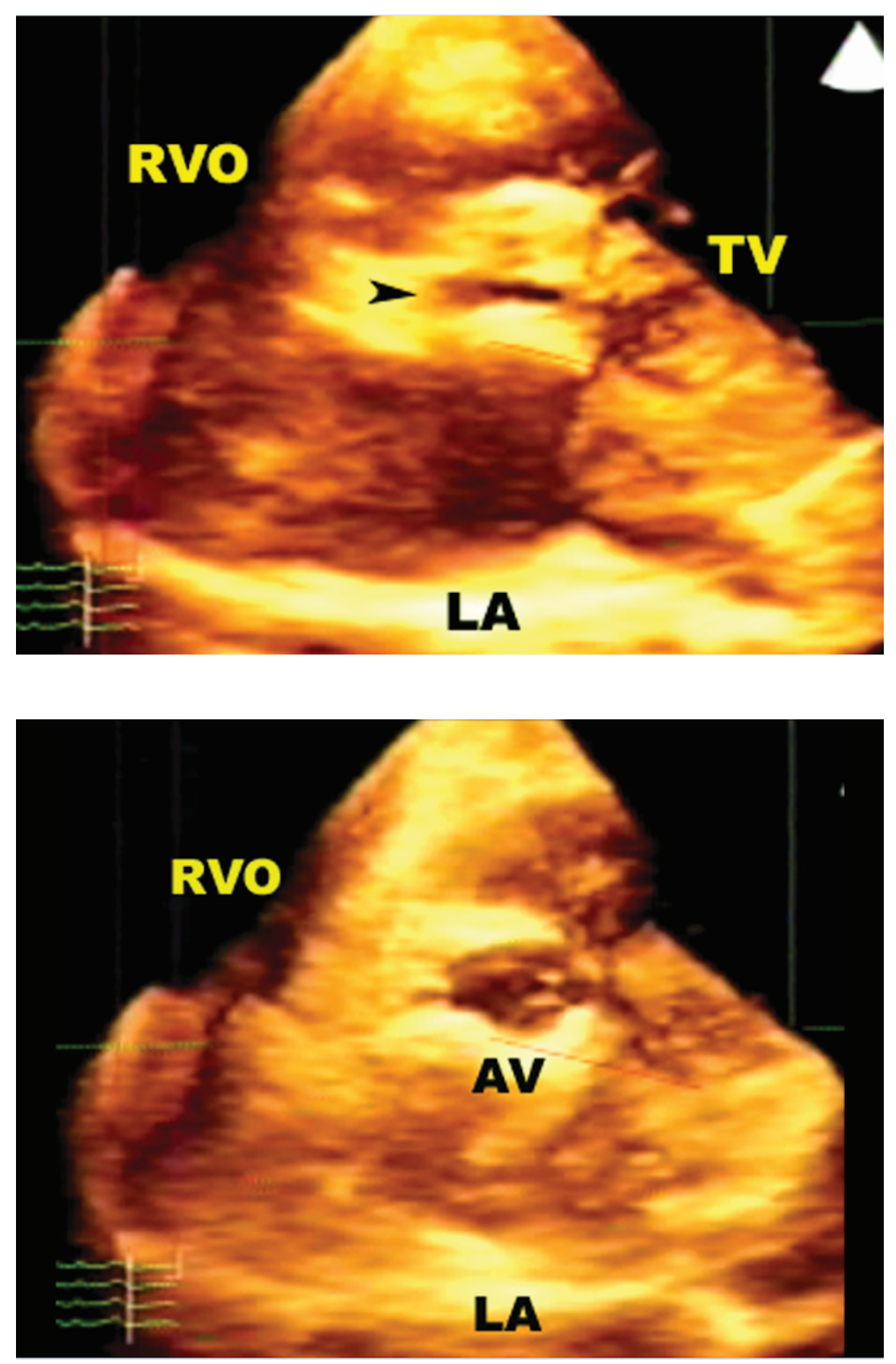


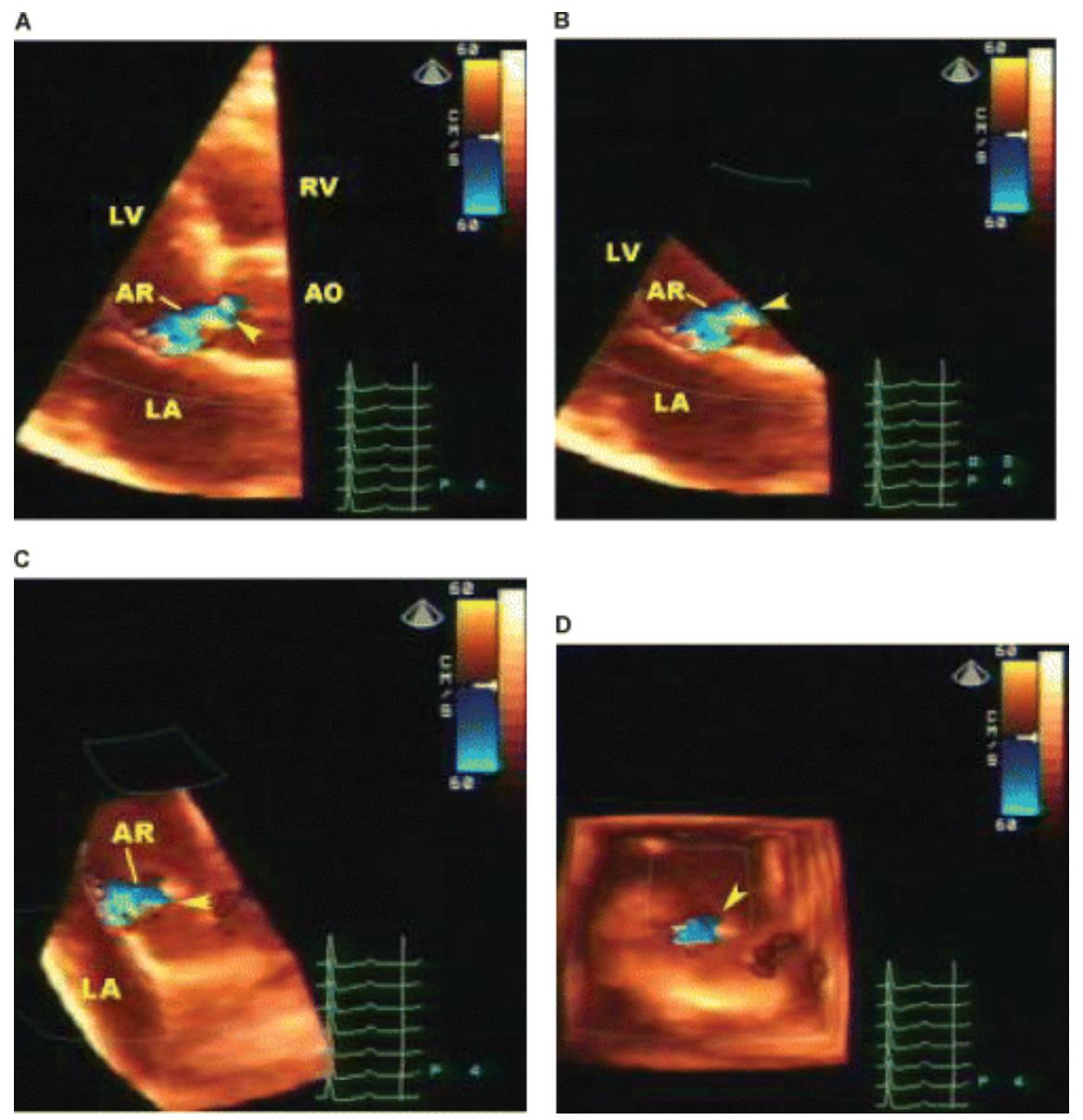



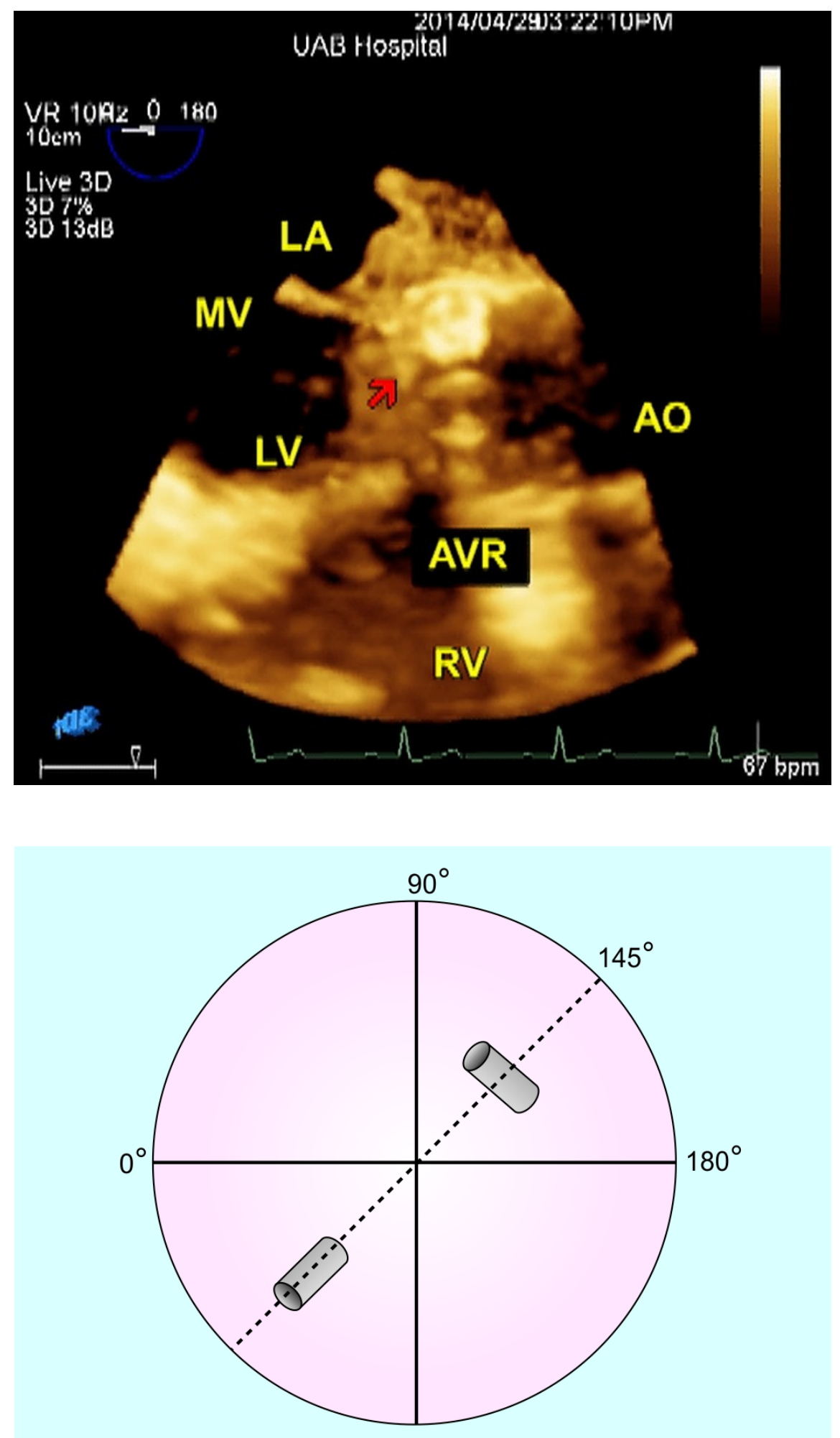

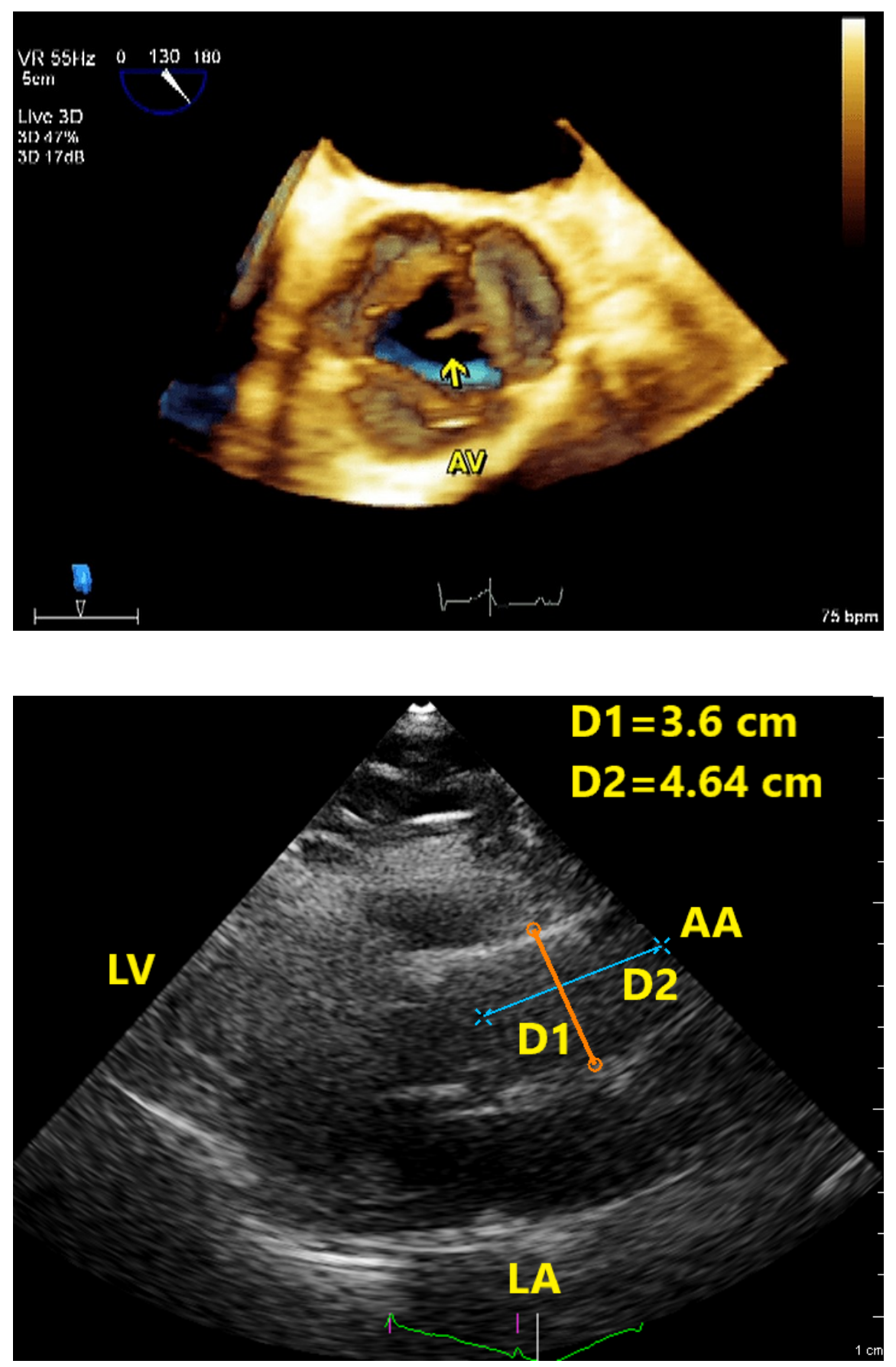

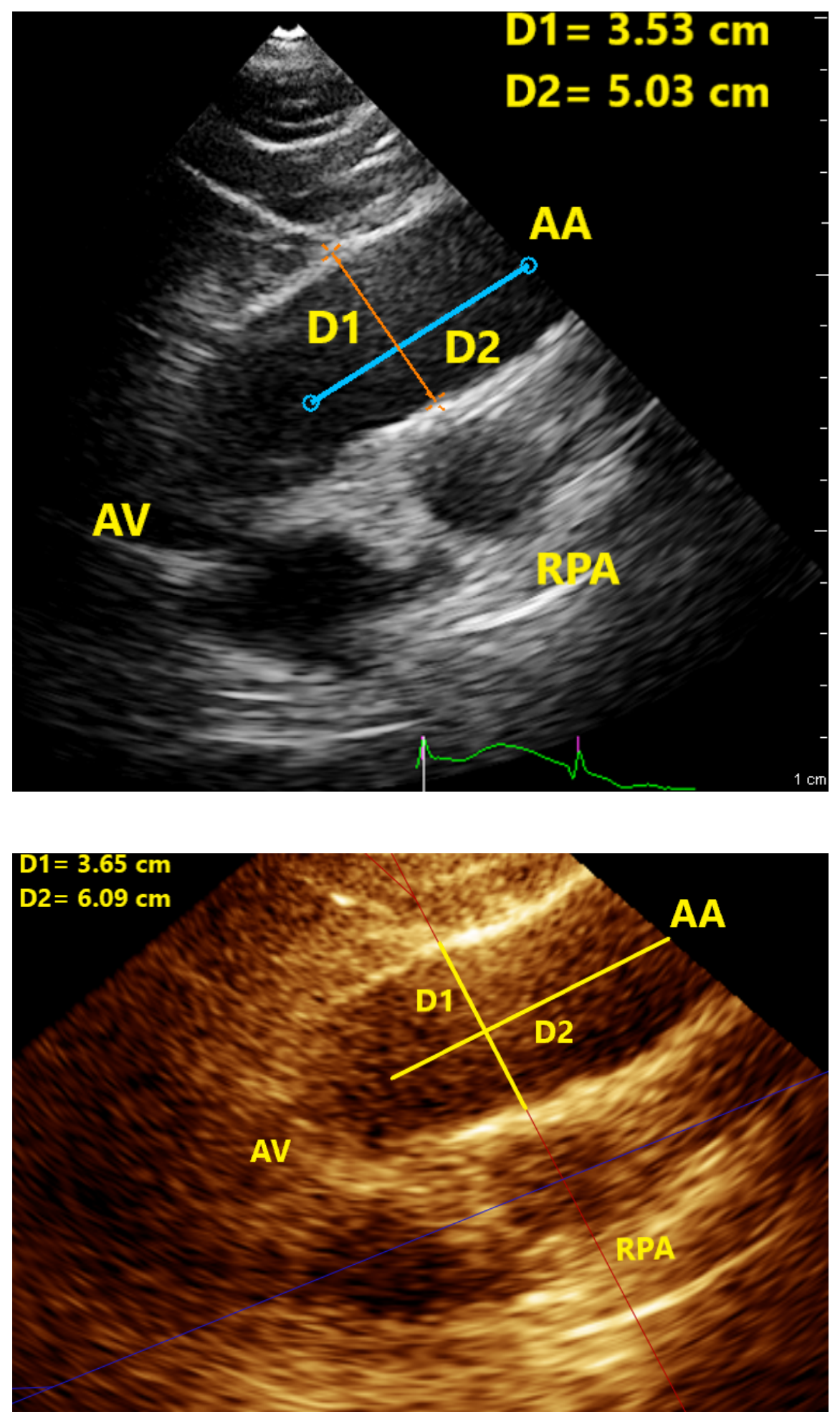

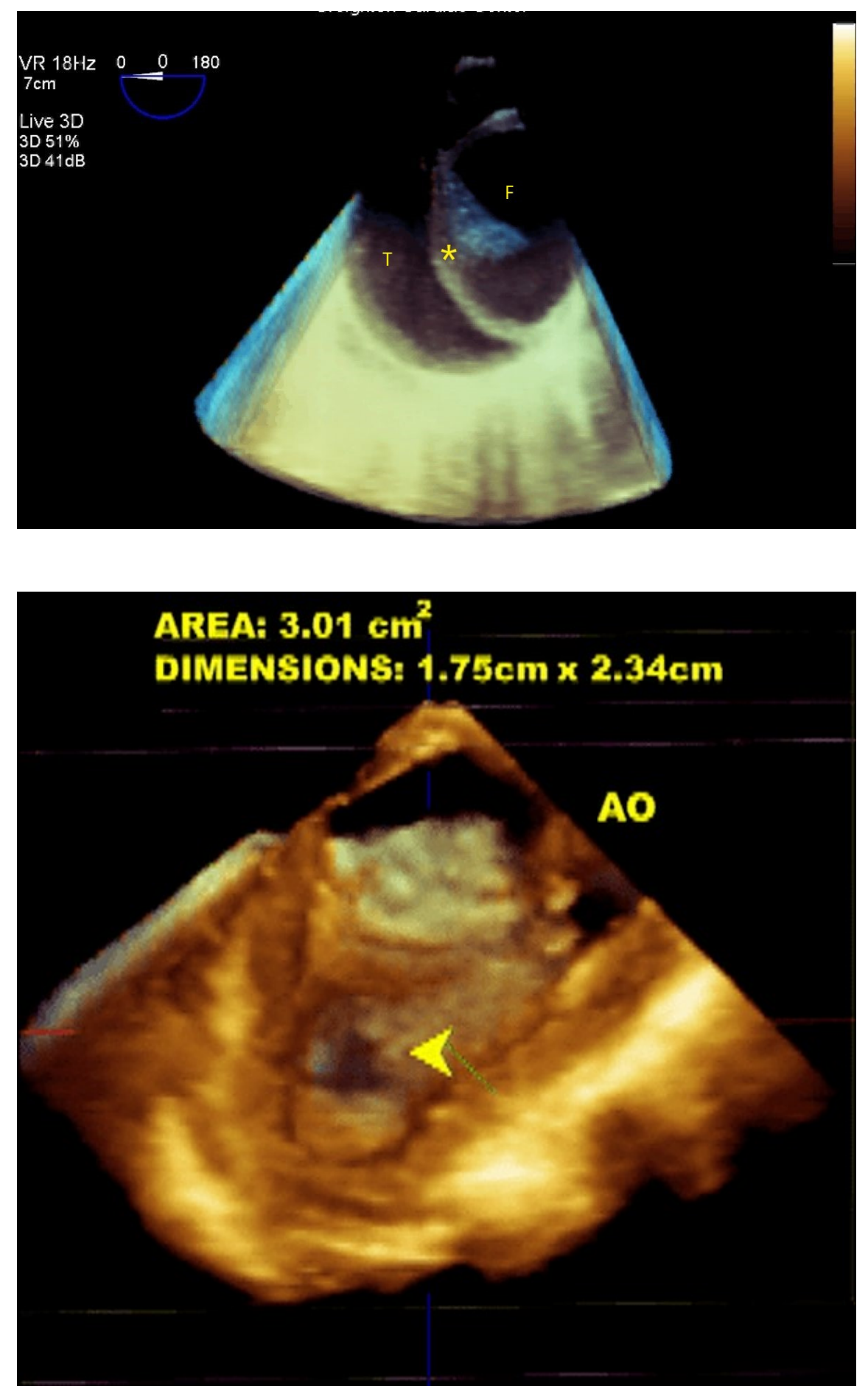
NPL

PL 


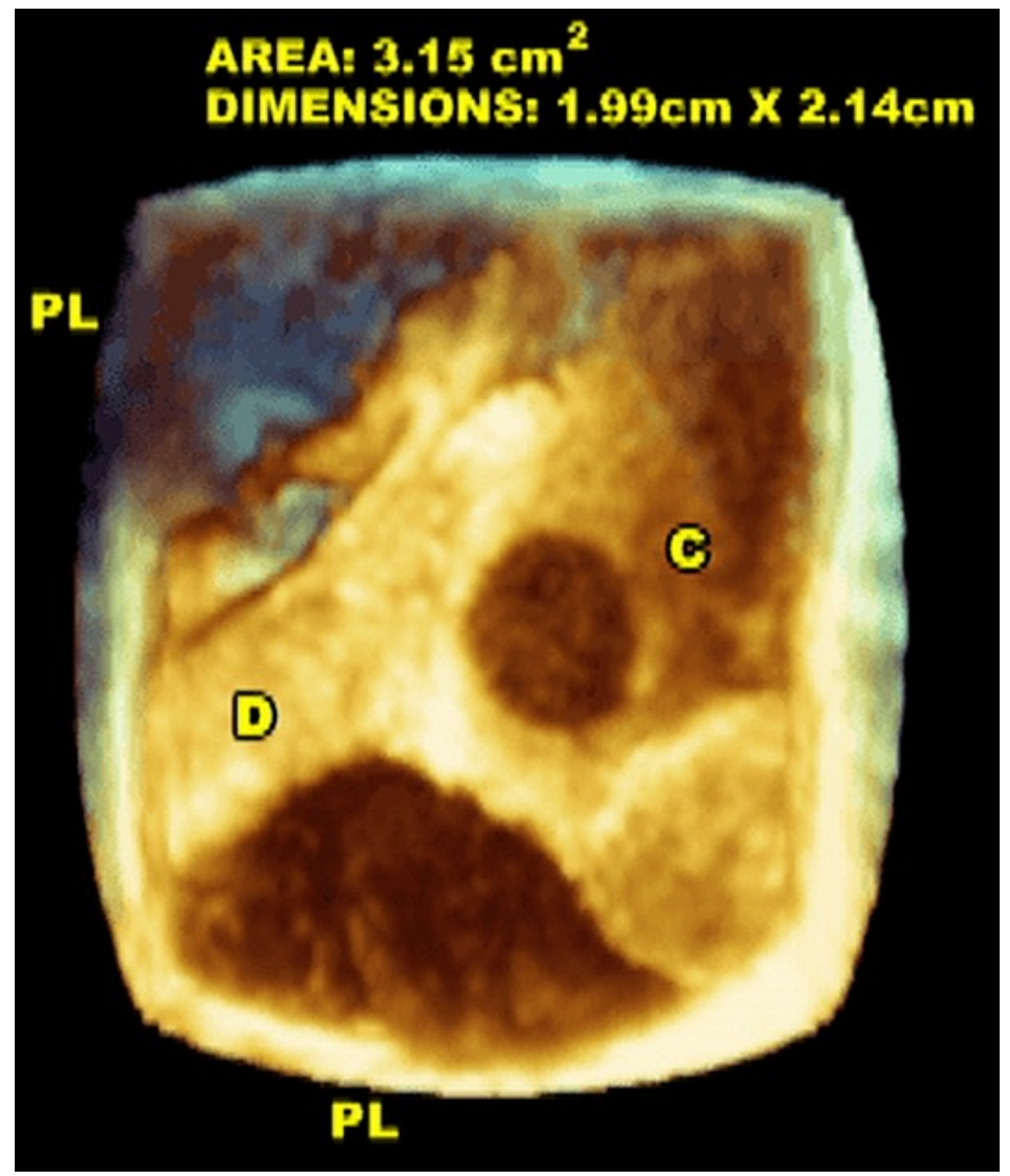



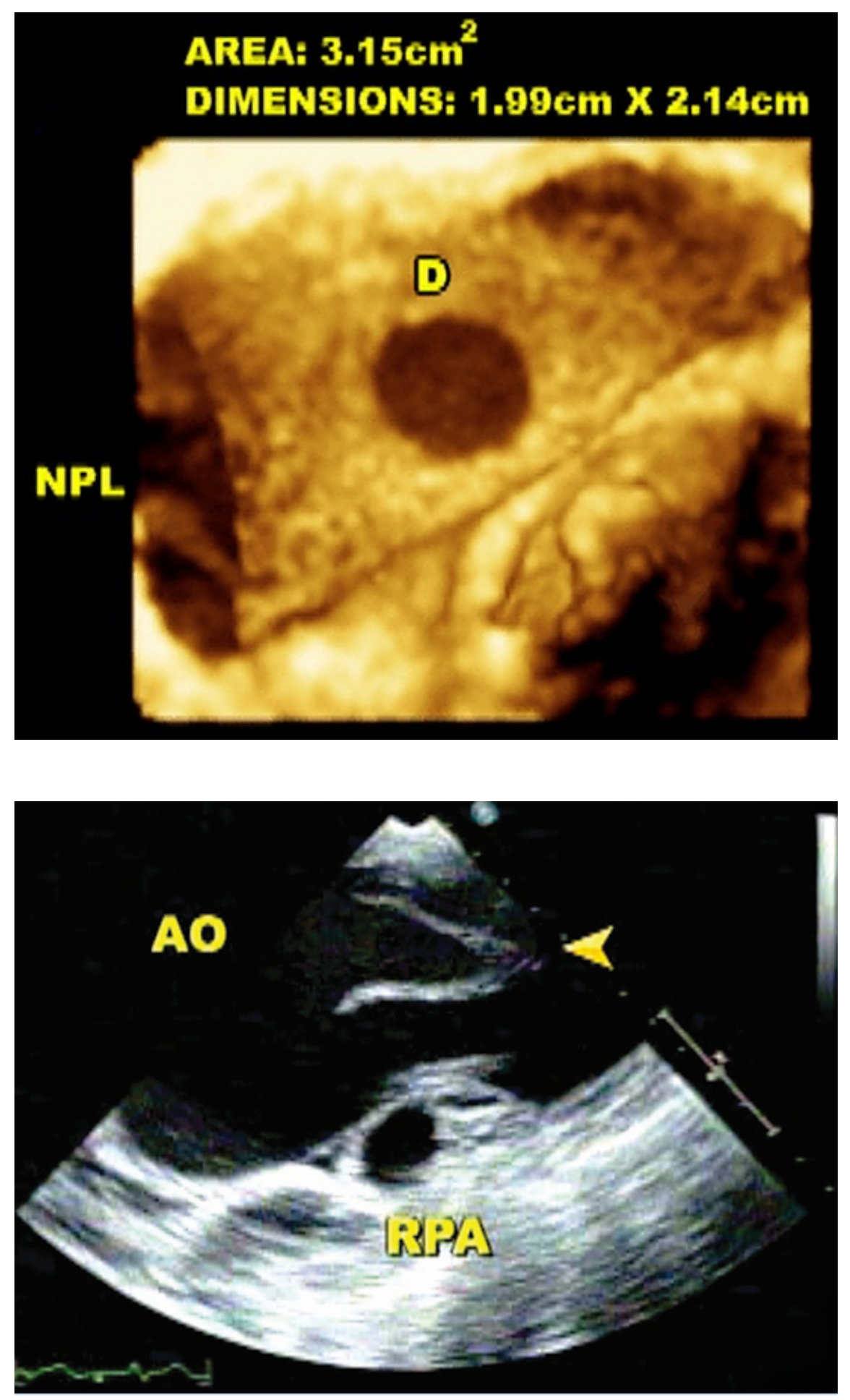


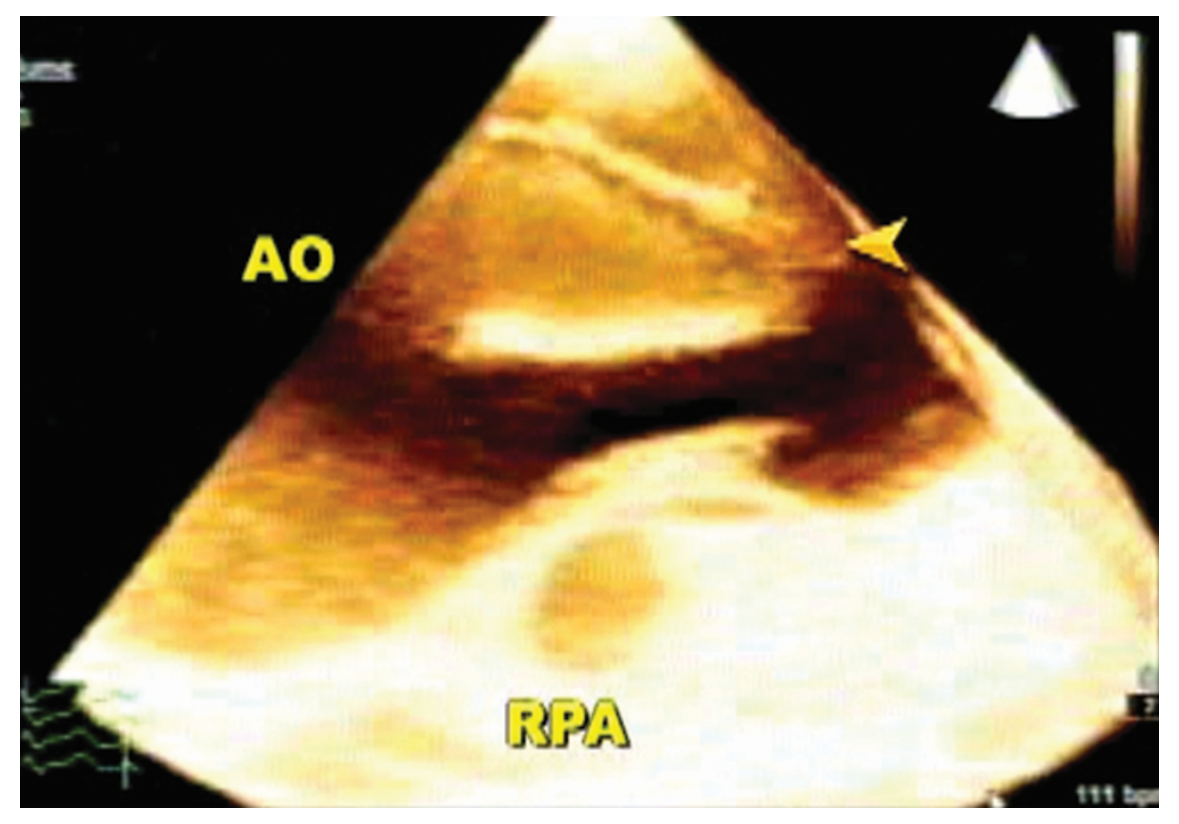

